\section{CTAS: a CT score to quantify disease activity in pulmonary sarcoidosis}

\section{Background A major gap in the management of sarcoidosis is the lack of accessible and objective methods to measure disease activity. Since $90 \%$ of patients have pulmonary involvement, we explored if a disease activity score based on thoracic CT scans could address this clinical issue.}

Methods High-resolution CT scans from 100 consecutive patients with sarcoidosis at a regional sarcoidosis service were scored for extent of CT abnormalities known to relate to granuloma or lymphocytic infiltration from published CT-pathological studies. These individual abnormality scores were then correlated against serum ACE, sIL-2R and change in FVC to identify CT abnormalities that reflect contemporaneous disease activity. The sum of these scores, or CT Activity Score (CTAS), was then validated against FVC response to treatment.

Findings $\mathrm{CT}$ extent scores for nodularity, ground-glass opacification, interlobular septal thickening and consolidation correlated significantly with at least one of the disease activity parameters and were used to form CTAS. CTAS was found to predict FVC response to treatment at 1 year and was highly reproducible between radiologists. An abbreviated CTAS (aCTAS), constructed from presence or absence of the four $\mathrm{CT}$ abnormalities, also showed significant correlation with FVC response to treatment. CTAS and aCTAS also correlated with response to treatment in the fibrotic subgroup.

Interpretation CTAS provides a concept for an objective and reproducible CT scoring method to quantify disease activity in sarcoidosis. The score can potentially be used to stratify patients according to disease activity, determine response to treatment and establish if fibrotic sarcoidosis is active.

A major gap in the management of sarcoidosis is the lack of accessible and objective methods to measure disease activity. ${ }^{1}$ This has contributed to difficulties in deciding when treatment should be started or discontinued, particularly in fibrotic disease, and has hampered the evaluation of costly treatments such as biologics or cytokine modulators. The most commonly reported end point in clinical trials for pulmonary sarcoidosis is change in lung function measurements. However, this does not detect reduction in disease activity after treatment, which, in sarcoidosis, can also occur without change in lung function.
Ninety per cent of patients with sarcoidosis have pulmonary involvement, and high-resolution CT (HRCT) scan of the lung is performed in nearly all patients for diagnostic purposes. In addition, CT-pathological studies have shown that typical abnormalities like bronchovascular nodularity, ground glass opacity and consolidation correlate to the presence of granuloma on open lung biopsies. $^{2-4}$ Nodularity also associates strongly with bronchoalveolar lavage (BAL) cell count and serum-soluble IL-2 receptor (sIL-2R) levels, ${ }^{4}$ and profusion of interlobular septal thickening (IST) correlates with BAL lymphocytosis. ${ }^{5}{ }^{6}$ We therefore explored if systematically measuring these HRCT abnormalities would allow quantification of disease activity in pulmonary sarcoidosis.

One hundred consecutive patients with pulmonary sarcoidosis from the Oxford Sarcoidosis Service who had HRCT scan performed from 2011 and fulfilling the diagnostic criteria proposed by WASOG were recruited. All patients were followed up for 1 year to record change in treatment and lung function. The study was approved by the South Central National Research Ethics Service Committee. Detailed study methods are found in online supplementary methods.

Thoracic HRCT scan and full pulmonary function testing were performed within 4 weeks of each other for all patients. Each HRCT was specifically scored for the presence, character and extent of sarcoidosisrelated HRCT abnormalities (ground glass opacification (GGO), IST, nodularity, conglomeration, consolidation and intrathoracic lymphadenopathy) using predefined criteria from either the Fleischner Society glossary of terms or predefined criteria from previous peer-reviewed publications (see online supplementary table S1-S3). In an unselected proportion of patientsserum sIL-2R levels $(n=21)$; serum ACE $(n=72)$ and lung function at baseline and 12 months after CT scan (regardless of treatment) $(n=40)$ were also performed. These parameters were chosen to reflect active macrophage and granuloma, T-cell activity and overall disease activity respectively. FVC change was measured as \% change after a year from baseline, to represent disease reversibility and therefore, a surrogate of activity.

Scoring of the HRCT scans was performed by a fellowship-trained consultant thoracic radiologist (RB). To evaluate reproducibility of the final composite score, the first 85 scans were selected for scoring by a second radiologist based at a district general hospital (EH).
Ninety-seven patients had complete CT analyses. Demographic data and specific clinical characteristics are shown in table 1 . One hundred per cent of patients had intrathoracic lymphadenopathy, 33\% showed presence of GGO, $73 \%$ nodularity, 34\% IST, 24\% conglomeration and $20 \%$ consolidation (further details in online supplementary table S4). Thirty-four (35\%) patients showed evidence of fibrosis. Four patients had evidence of pulmonary hypertension on CT scan, confirmed on right heart pressure assessment by echocardiography.

Extent scores for nodularity, IST, consolidation and GGO but not conglomeration or lymphadenopathy correlated with at least one of the surrogates of disease activity (see online supplementary table S5). Based on these findings, we excluded conglomeration and lymphadenopathy and took the sum of the scores of GGO, IST, consolidation and nodularity as the composite score for disease activity. We termed this the 'CT Activity Score' or 'CTAS'. The CTAS score in our cohort ranged from 0 to 58 , with a median of 10 (IQR 0-19) (see online supplementary figure S1A). Patients with fibrosis had higher CTAS compared with non-fibrotic ((median of 15.8 (IQR 8.5-22.0) vs 9.8 (4.0-15.0); $\mathrm{p}<0.001$, Mann-Whitney U test) (see online supplementary figure

Table 1 Demographic data for patient cohort

\begin{tabular}{ll}
\hline $\mathbf{n}$ & $\mathbf{9 7}$ \\
\hline Age range (years) & $24-79$ \\
Age mean (SD) & $51(13)$ \\
FEV $_{1}$, range & $32-124 \%$ \\
FEV $_{1}$, mean (SD) & $85(20) \%$ \\
FVC, range & $37-135 \%$ \\
FVC, mean (SD) & $98(19) \%$ \\
DLCO, range & $26-139 \%$ \\
DLCO, mean (SD) & $80(21) \%$ \\
Gender (F) & $42(44 \%)$ \\
Current smoker & $8(8 \%)$ \\
Afro-Caribbean & $6(6 \%)$ \\
Asian (sub-Indian continent) & $3(3 \%)$ \\
Scadding stage 1/2/3/4 & $20 / 49 / 9 / 19$ \\
Lung involvement & $97(100 \%)$ \\
Skin involvement & $8(8 \%)$ \\
Liver involvement & $7(7 \%)$ \\
Cardiac involvement & $7(7 \%)$ \\
CNS involvement & $2(2 \%)$ \\
Bone involvement & $2(2 \%)$ \\
Eye involvement & $10(10 \%)$ \\
\hline Pulmoar &
\end{tabular}

Pulmonary function test refers to \% predicted for age, gender and height. Unless stated, \% in parentheses refer to $\%$ of cohort.

CNS, central nervous system. 
S1B); and CTAS was higher with worse lung function (see online supplementary figure 1C-E). Examples of CT scans showing divergent CTAS are shown in online supplementary figure S2.

To provide a validation of the relationship of CTAS to disease activity, we examined if CTAS was able to predict the size of response to treatment. We reasoned that if CTAS reflected disease activity, then, the higher the CTAS value, the greater the ability of lung function (FVC) to improve with treatment. Twenty-nine patients went on to start or increase treatment specifically for their lung disease within 4 weeks of HRCT scanning. FVC values at 12 months ( \pm 8 weeks) after treatment were compared with pretreatment. We found that CTAS predicted the size of FVC response to treatment at 1 year $(b=0.42$ (CI 0.05 to 0.79$) ; r^{2}=0.17$, $\mathrm{p}=0.03$ ) (figure 1A). This was also the case for the fibrotic subgroup, where 20 of the 34 patients received treatment $(\mathrm{b}=0.32 \quad$ (CI 0.0 to 0.6$) ; \mathrm{r}^{2}=0.20$, $\mathrm{p}=0.04$ ) (figure 1B).

Comparing the CTAS with any one CT parameter (GGO, nodularity, consolidation and IST), we found only GGO and consolidation to predict treatment response $(p=0.04$ and 0.05 , respectively), and at a weaker certainty than CTAS $(p=0.03)$. Therefore, the composite measure of CTAS provides the strongest correlation with FVC response after treatment.

We also examined an abbreviated CTAS (aCTAS) comprising the sum of presence (1) or absence (0) of each of the four CT features (GGO, nodularity, IST and consolidation) and found that this produced similar correlation outcomes to CTAS for lung function and response to treatment (see figure 1C and D and online supplementary figure S3).

We found a strong correlation between radiologists for the CTAS $(r=0.91$; $\mathrm{p}<0.0001$; Pearson's correlation test; $\mathrm{n}=85$ ), and Bland-Altman analysis confirmed that there was no systematic deviation in measurements over the range of values (see online supplementary figure S4). There was no difference in aCTAS between the two radiologists $(r=1.0$; $\mathrm{n}=85)$.

We propose CTAS as a promising concept for the measurement of disease activity in pulmonary sarcoidosis. Our study was limited in size but is the first description of a numerical CT score for the extent of disease activity in pulmonary sarcoidosis. The validation component was limited to patients who were started on treatment during the year $(n=29)$ and will benefit from further evaluation with a bigger cohort. Measurements of disease severity on HRCT (but not activity) have been previously published. ${ }^{7-10}$ CTAS is specifically designed to reflect the extent of disease activity regardless of the level of lung function impairment and fibrosis. Indeed, CTAS and aCTAS could be particularly useful in aiding decisions on whether to treat fibrotic sarcoidosis. A CT scan showing volume loss and established fibrosis has often been termed 'burnt out' fibrosis but may contain areas of active disease that may be driving the fibrotic process. We propose that a conclusion of 'active' or 'inactive' disease (with aCTAS), based on the absence or presence of any of these four CT abnormalities, could be a useful addition to an HRCT report for pulmonary sarcoidosis. A validation study will strengthen and possibly refine the score and provide an opportunity to bring this score into clinical practice.

Rachel Benamore, ${ }^{1}$ Yvonne R Kendrick, ${ }^{2,3}$ Emmanouela Repapi, ${ }^{4}$ Emma Helm, ${ }^{5}$ Suzanne L Cole, ${ }^{3}$ Stephen Taylor, ${ }^{4}$ Ling-Pei $\mathrm{Ho}^{2,3}$

${ }^{1}$ Thoracic Imaging Department, Churchill Hospital, Oxford, UK

${ }^{2}$ Oxford Interstitial Lung Disease Service, Oxford Centre for Respiratory Medicine, Churchill Hospital, Oxford, UK ${ }^{3}$ MRC Human Immunology Unit, Weatherall Institute of Molecular Medicine, University of Oxford, Oxford, UK

${ }^{4}$ Computational Biology Research Group, Weatherall Institute of Molecular Medicine, University of Oxford, Oxford, UK

${ }^{5}$ Radiology Department, University Hospitals Coventry and Warwickshire NHS Trust, Coventry, UK

Correspondence to Ling-Pei Ho, Oxford Interstitial Lung Disease Service, Oxford Centre for Respiratory Medicine, Churchill Hospital, Oxford OX3 7LJ, UK; Lingpei.ho@imm.ox.ac.uk

- Additional material is published online only. To view please visit the journal online (http://dx.doi.org/10. 1136/thoraxjnl-2016-208833).

RB and YRK are joint first authors.

Contributors YRK contributed to design, writing and analysis of data. RB scored the $\mathrm{CT}$ and contributed to design and discussions of the data. EH scored the second set of CTs and contributed to discussion of the manuscript. ER performed the statistical design, analysis and contributed to the design of the study.

ST contributed to statistical analysis. SLC performed some of the serum analyses. L-PH conceived the idea, designed, analysed and wrote the paper.

Funding The study was funded by the NIHR Biomedical Research Centre at Oxford.

Competing interests None declared.

Ethics approval South Central National Research Ethics Service Committee.

Provenance and peer review Not commissioned; externally peer reviewed.

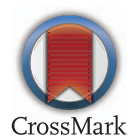

To cite Benamore R, Kendrick YR, Repapi E, et al. Thorax 2016;71:1161-1163.

Received 26 April 2016

Revised 16 June 2016

Accepted 1 July 2016

Published Online First 27 July 2016

Thorax 2016:71:1161-1163.

doi:10.1136/thoraxjnl-2016-208833

\section{REFERENCES}

1 Valeyre D, Prasse A, Nunes $H$, et al. Sarcoidosis. Lancet Respir Med 2014;383:1155-67.

2 Spagnolo P, Sverzellati N, Wells AU, et al. Imaging aspects of the diagnosis of sarcoidosis. Eur Radiol 2014;24:807-16.

3 Leung AN, Brauner MW, Caillat-Vigneron N, et al. Sarcoidosis activity: correlation of HRCT findings with those of $67 \mathrm{Ga}$ scanning, bronchoalveolar lavage, and serum angiotensin-converting enzyme assay. J Comput Assist Tomogr 1998;22:229-34. for all patients $(A$ and $C$ ) and for the fibrotic subgroup ( $B$ and $D) . r$ and $p$ Values shown are from linear regression calculations. Spearman correlation values are $(A) r=0.40, p=0.03$; $(B) r=0.35$, $p=0.13$; (C) $r=0.42, p=0.02$; and (D) $r=0.34, p=0.13$. 
4 Nishimura $\mathrm{K}$, Itoh $\mathrm{H}$, Kitaichi $\mathrm{M}$, et al. Pulmonary sarcoidosis: correlation of $\mathrm{CT}$ and histopathologic findings. Radiology 1993;189:105-9.

5 Nishimura $\mathrm{K}$, Itoh $\mathrm{H}$, Kitaichi $\mathrm{M}$, et al. CT and pathological correlation of pulmonary sarcoidosis. Semin Ultrasound CT MR 1995;16:361-70.

6 Oberstein A, von Zitzewitz H, Schweden F, et al. Non invasive evaluation of the inflammatory activity in sarcoidosis with high-resolution computed tomography. Sarcoidosis Vasc Diffuse Lung Dis 1997;14:65-72.

7 Remy-Jardin M, Giraud F, Remy J, et al. Pulmonary sarcoidosis: role of $\mathrm{CT}$ in the evaluation of disease activity and functional impairment and in prognosis assessment. Radiology 1994;191:675-80.

8 Drent M, De Vries J, Lenters M, et al. Sarcoidosis: assessment of disease severity using HRCT. Eur Radiol 2003;13:2462-71.

9 Zappala CJ, Desai SR, Copley SJ, et al. Accuracy of individual variables in the monitoring of long-term change in pulmonary sarcoidosis as judged by serial high-resolution CT scan data. Chest. 2014;145:101-7.

10 Prasse A, Katic C, Germann M, et al. Phenotyping sarcoidosis from a pulmonary perspective. $\mathrm{Am}$ J Respir Crit Care Med 2008;177:330-6. 